\title{
TENDENCIAS EN LA PRECIPITACIÓN, POR DISTINTOS INPUTS, EN EL SECTOR CENTRAL DE LA PENÍNSULA IBÉRICA (1958-2008)
}

\author{
María José Estrela $^{\mathrm{a}}$, Javier Mirób ${ }^{\text {, Francisco } \text { Pastor }^{\mathrm{b}} \text { y Millán Millán }}{ }^{\mathrm{b}}$
}

\section{RESUMEN}

Estudios previos llevados a cabo por el Grupo de Meteorología de la Fundación CEAM para el análisis de las tendencias de la precipitación según origen genético, han permitido detectar tendencias negativas en las precipitaciones por advecciones atlánticas y advecciones continentales septentrionales, en el Este de la Península Ibérica, durante los últimos 50 años. Estas tendencias negativas han tenido una repercusión para áreas del interior del Este de la Península Ibérica que no han tenido en el litoral, debido al mayor peso que el input 'atlánticas' tiene en el interior. El objetivo de este trabajo es analizar si se detectan las mismas tendencias descendentes en las precipitaciones de origen atlántico en el sector central de la Península Ibérica, dado que éste es el ámbito vecino al sector más interior de la Comunidad Valenciana, área donde adquieren mayor importancia las precipitaciones de origen atlántico. Para ello se ha trabajado en dos sectores, uno al Norte del Sistema Central y otro al Sur del mismo, utilizando la misma metodología desarrollada por el Grupo de Meteorología-Climatología de desagregación sinóptica. Los resultados, no sólo confirman la hipótesis al descenso de estas precipitaciones, sino que además muestran tendencias bastante generalizadas al descenso de los totales de la precipitación, sobre todo para el sector localizado al Norte del Sistema Central (meseta Norte).

Palabras clave: inputs de precipitación, tendencias, Sistema Central, advecciones atlánticas, desagregación de la precipitación.

Laboratorio de Meteorología-Climatología, Unidad Mixta CEAM-UVEG (Universidad de Valencia). Departament de Geografia Física, Universitat de Valencia, Avda. Blasco Ibañez 28, 46010 Valencia, Spain.

Laboratorio de Meteorología-Climatología, Unidad Mixta CEAM-UVEG. Fundación Centro de Estudios Ambientales del Mediterráneo, Área de Meteorología-Climatología, Parque Tecnológico, Calle Charles R. Darwin 14, 46980 Paterna, Valencia, Spain. 


\begin{abstract}
Previous papers from the CEAM Group in relation to the analysis of precipitation by meteorological origin, have permitted the detection of negative trends in the precipitation for frontal systems and continental advections (Northern) in the last 50 years. These negative trends have had important repercussions in the inland areas of the Valencia Region and not on the coast. The objective of this work is to analyze if the same decreasing trends can be detected in the precipitations from the Atlantic origin in areas of the interior of the Iberian Peninsula. In order to do that we have worked in two sectors, the North and the South of the Central System. We worked with the same methodology of desaggregation. The results not only confirm the hypothesis of precipitation decrease but show generalized trends in decreasing totals precipitations, especially in the North of the Central System.
\end{abstract}

Key words: inputs of precipitation, trends, Central System, atlantic frontal systems, disaggregate the precipitation series.

\title{
1. Introducción y objetivos
}

En un trabajo previo (Miró et al., 2010) se ha realizado un análisis comparativo de la precipitación entre los dominios hidrográficos del Segura y del Júcar, a partir del uso de un método de desagregación de la precipitación según su origen genético. En el presente trabajo los autores van a aplicar este método de desagregación de la precipitación al sector central de la Península Ibérica. Tradicionalmente, se ha considerado que las precipitaciones de procedencia atlántica tienen un peso muy marcado en el sistema hídrico de la vertiente atlántica peninsular. Sin embargo, no existen muchos trabajos que hayan analizado su peso en el sistema frente a precipitaciones de otras procedencias, tales como convectivas o de origen mediterráneo. Y sin embargo, podemos considerar que este análisis es fundamental si tenemos presente los resultados ya obtenidos para las precipitaciones de distinto origen de recarga para la Comunidad Valenciana (Estrela et al., 2004 y 2006; Millán et al., 2005 y 2006; Miró et al., 2006). De confirmarse este mismo marcado descenso de las de origen atlántico para otras áreas del interior, tendría implicaciones muy significativas, dado que su peso relativo es mucho mayor.

Por tanto, ahora el propósito es conocer si las pautas observadas en los diferentes inputs de precipitación, analizados para diversas áreas de la vertiente mediterránea de la Península Ibérica (cuencas del Segura y Júcar), se repiten en otros sectores más al interior peninsular. Para ello se ha llevado a cabo el mismo análisis de la precipitación desagregada según su origen genético. El área de estudio se localiza al Norte y Sur del Sistema Central. El análisis está basado en el método desarrollado por los autores (Millán et al., 2005), aunque en este caso se ha mejorado el proceso de desagregación por inputs haciéndolo automático con el objeto de eliminar la parte de subjetividad que llevaba implícito, así como de facilitar la ampliación tanto espacial como temporal del análisis (Miró et al., 2010). El análisis abarca el período 1958-2008, aunque para determinados análisis se han diferenciado dos etapas (1958-1978 y 1978-2008), a fin de detectar tendencias y cambios estructurales en la precipitación.

\section{Metodología}

Se ha trabajado con la metodología desarrollada en Miró et al., (2010). En resumen, el método de trabajo consta de los siguientes procesos: 
1. Obtención y catalogación de los inputs sobre una base diaria, con la adscripción de todos los días disponibles desde 1958 hasta 2008 a un input pluviométrico en particular. Esos son: 'Atlántica', 'Retroceso', 'Retroceso seca' y 'Convectiva'. En la figura 1 se indican las procedencias de los diferentes inputs.

2. Elección del marco territorial y selección de un grupo de estaciones representativas para cada sector, que cubran totalmente el periodo de 1958 a 2008 con un porcentaje superior al $95 \%$ de datos válidos.

3. Realización del análisis de tendencias, sobre los valores anuales de precipitación (totales y de cada input en particular), utilizando los siguientes métodos: El test de Mann-Kendall (Mann, 1945; Kendall, 1975), la estimación Sen de Pendiente (Sen, 1968), y el Análisis de Espectro Singular o SSA (Elsner y Tsionis, 1996).

Se ha preparado para la catalogación diaria de inputs una malla de datos del NCEP, que comprende las latitudes entre $45^{\circ} \mathrm{N}$ y $37.5^{\circ} \mathrm{N}$ y las longitudes entre $10^{\circ} \mathrm{W}$ y $0^{\circ} \mathrm{E}$ (Fig. 1). Los criterios de cálculo con los que se trabaja son comunes para con el caso de la malla utilizada en Miró et al., 2010, por lo que los resultados para los diferentes inputs son perfectamente comparables entre ambos ámbitos.

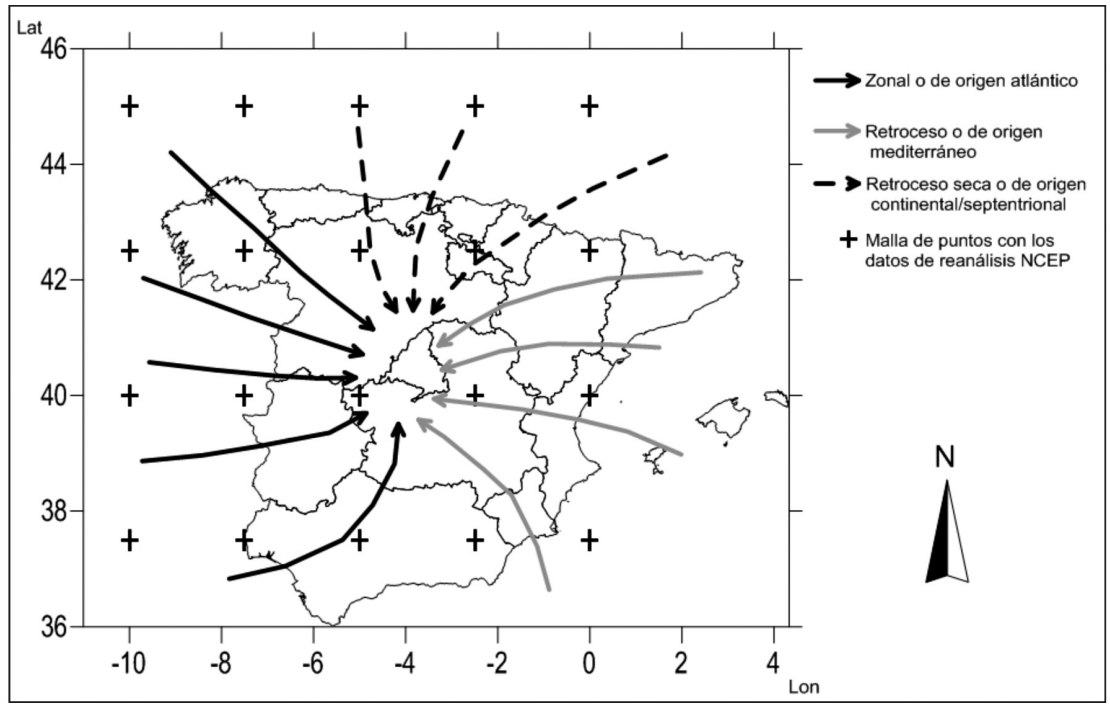

FIgURA 1. Flujos que componen cada input, y malla de datos de reanálisis del NCEP utilizada para el cálculo del flujo dominante.

Para el caso peninsular, dentro del input 'retroceso seca', ahora ganan peso las advecciones procedentes del Mar Cantábrico, que realmente tienen un escaso recorrido continental. Sin embargo, el objeto de este input para estudios en el ámbito europeo de la cuenca del Mediterráneo (no sólo su parte peninsular), era la de incluir las advecciones puramente continentales (procedentes del interior de Europa). No obstante, consideramos aquí el uso del mismo patrón de flujos, por un lado, porque el efecto de sotavento de la Cordillera 
Cantábrica es patente, y por otro, el peso relativo de este input sigue siendo igualmente bajo, en todos los casos, para el centro peninsular con respecto a los de la zona levantina. Y también, el valor comparativo que proporciona aconseja conservar la misma estructura original de inputs.

Se han seguido criterios geográficos e hidrográficos en la delimitación de las áreas de estudio. El Sistema Central es una barrera montañosa que muestra un comportamiento desigual de la precipitación según sea la vertiente Norte o Sur, y al mismo tiempo proporciona un carácter climatológicamente unitario a lo largo de cada una de sus dos vertientes. A esto se une la pertenencia del área a dos cuencas hidrográficas distintas, la del Duero y la del Tajo. Se ha trabajado con un total de cinco estaciones en el sector Norte del Sistema Central, y cuatro estaciones en el sector Sur, que cubren plenamente el período analizado (Fig. 2). Como serie de testigo (análisis de tendencias) se utiliza el promedio de las series de las estaciones que conforman cada sector, lo que aún redunda en una mayor inhibición de cualquier posible no homogeneidad no detectada en una serie individual.

Las cinco estaciones del sector Norte son: Burgos (Villafría), Navacerrada (Puerto), Riaza (Segovia), Salamanca (Matacán) y Pantano de Sta. Teresa (Salamanca). Y las del sector Sur son: Molina de Aragón (Guadalajara), Madrid (Retiro), Berzocana (Cáceres) y Badajoz (Talavera 'Base aérea'). Todas pertenecen a la red de estaciones del AEMET.

Finalmente, el análisis de tendencias se aplicará sobre la base de los volúmenes anuales de precipitación entre 1958-2008, tanto para los totales anuales, como para la desagregación en inputs. Al igual que en el caso precedente en MIRÓ et al. 2010, se ha utilizado la macro 'Makesens' (Salmi, T. et al., 2002), y el software 'Caterpillar-SSA' (Alexandrov, 2005 y 2006).

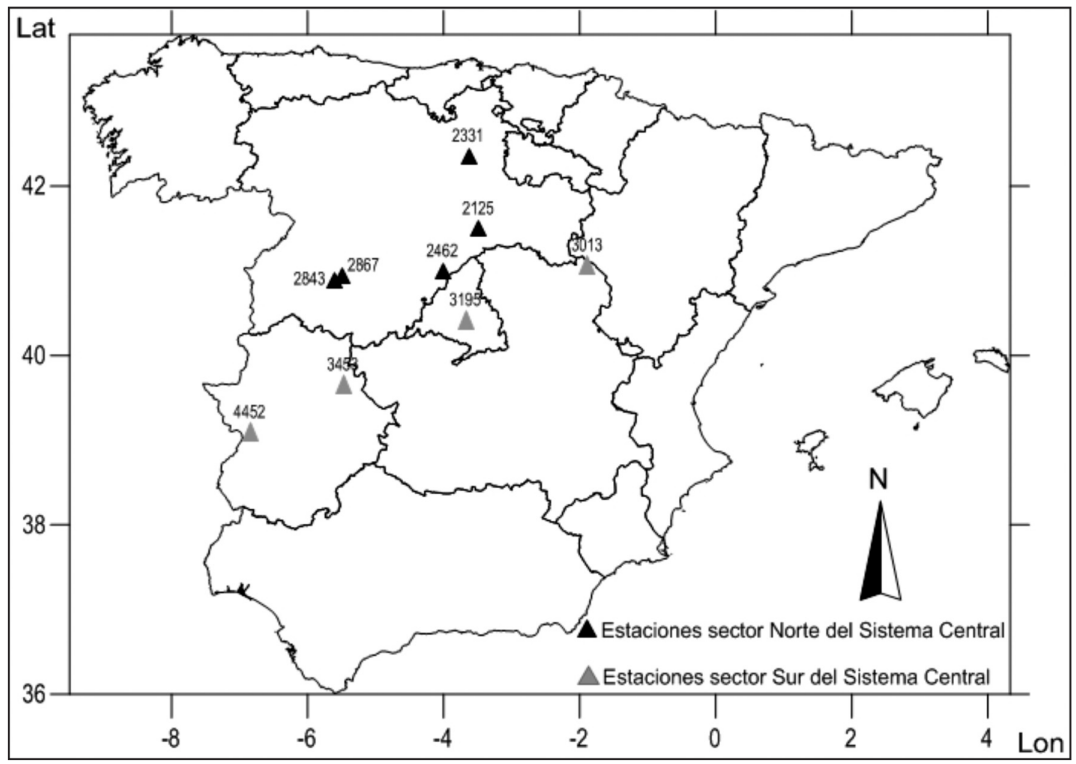

FIGURA 2. Localización del total de estaciones utilizadas para cada ámbito geográfico, y sus códigos según la catalogación de la AEMET. 


\section{Resultados}

\subsection{Análisis de las tendencias}

Tabla 1

TEST DE MANN-KENDALL Y DE LA ESTIMACIÓN SEN DE PENDIENTE PARA TODAS LAS ÁREAS DE ESTUDIO E INPUTS

\begin{tabular}{|l|r|r|r|r|r|r|r|r|r|r|}
\hline & \multicolumn{4}{l|}{ Mann-Kendall trend } & \multicolumn{2}{l|}{ Sen's slope estimate } \\
\hline Series & Comienza & Acaba & n & Test Z & Signific. & Q & Qmin99 & Qmax99 & Qmin95 & Qmax95 \\
\hline Norte SC, total & 1958 & 2008 & 51 & -2.84277 & $* *$ & -4.123 & -7.824 & -0.392 & -6.797 & -1.2111 \\
\hline Sur SC, total & 1958 & 2008 & 51 & -1.66511 & + & -2.446 & -6.18 & 1.4201 & -5.175 & 0.46453 \\
\hline Norte SC, atlántica & 1958 & 2008 & 51 & -2.06304 & $*$ & -2.385 & -5.34 & 0.6726 & -4.33 & -0.0943 \\
\hline Sur SC, atlántica & 1958 & 2008 & 51 & -1.3158 & & -1.311 & -4.65 & 1.9484 & -3.824 & 1.06492 \\
\hline Norte SC, retroceso seca & 1958 & 2008 & 51 & -2.30671 & $*$ & -0.568 & -1.244 & 0.0592 & -1.064 & -0.0865 \\
\hline Sur SC, retroceso seca & 1958 & 2008 & 51 & -1.66511 & + & -0.222 & -0.617 & 0.1217 & -0.518 & 0.04743 \\
\hline Norte SC, retroceso & 1958 & 2008 & 51 & -0.4386 & & -0.256 & -1.364 & 0.8317 & -1.112 & 0.57158 \\
\hline Sur SC, retroceso & 1958 & 2008 & 51 & -0.51172 & & -0.272 & -1.434 & 0.9506 & -1.146 & 0.59946 \\
\hline Norte SC, convectiva & 1958 & 2008 & 51 & -1.83562 & + & -0.454 & -1.186 & 0.2389 & -0.996 & 0.03449 \\
\hline Sur SC, convectiva & 1958 & 2008 & 51 & -2.24173 & $*$ & -0.409 & -0.919 & 0.0572 & -0.818 & -0.049 \\
\hline
\end{tabular}

\subsubsection{Sector Norte del Sistema central}

Para este sector los totales anuales ya muestran una tendencia indiscutible al descenso de la precipitación. El test de Mann-Kendall (Tabla 1) muestra una tendencia muy negativa, de casi -3, y además con un alto nivel de significación, superior al $99 \%$. Igualmente, la pendiente Sen, con un valor inferior a -4 , muestra intervalos de confianza siempre de carácter negativo. Estos datos, ya de por si concluyentes, se ven además apoyados por la línea de tendencia extraída mediante el SSA (Fig. 3), muy descendente, al menos hasta mediados de los 90, y con una perdida en el volumen de precipitación próxima al $25 \%$ entre el principio y el fin de la serie. Este dato es muy significativo tratándose de totales absolutos de precipitación.

En el análisis desagregado de la precipitación por inputs se aprecia un descenso de los inputs 'atlánticas', 'retroceso seca' y 'convectiva'. Sólo las situaciones de 'retroceso' de origen o recarga mediterránea no muestran esta tendencia.

Las precipitaciones de origen atlántico muestran tendencia negativa significativa en el test de Mann-Kendall (con significación superior al 95\%), sumándose igualmente la estimación Sen con valores aceptables en los intervalos de confianza (Tabla 1). La tendencia extraída mediante SSA (Fig. 4) apoya este escenario negativo, más marcado entre el inicio de la serie y mediados de los 90 , con un comportamiento posterior más plano. Y una pauta similar presenta el input 'retroceso seca', con buenos niveles de significación en el test de Mann-Kendall, que se ven reforzados en esta tendencia negativa por el SSA (Fig. 6).

Con relación al input 'convectivas' también se aprecia una clara tendencia al descenso similar a la que los autores ya habían detectado en sectores del interior de la Comunidad Valenciana (Estrela, 2006), resultado de una pérdida de las tormentas tardo-primaverales 


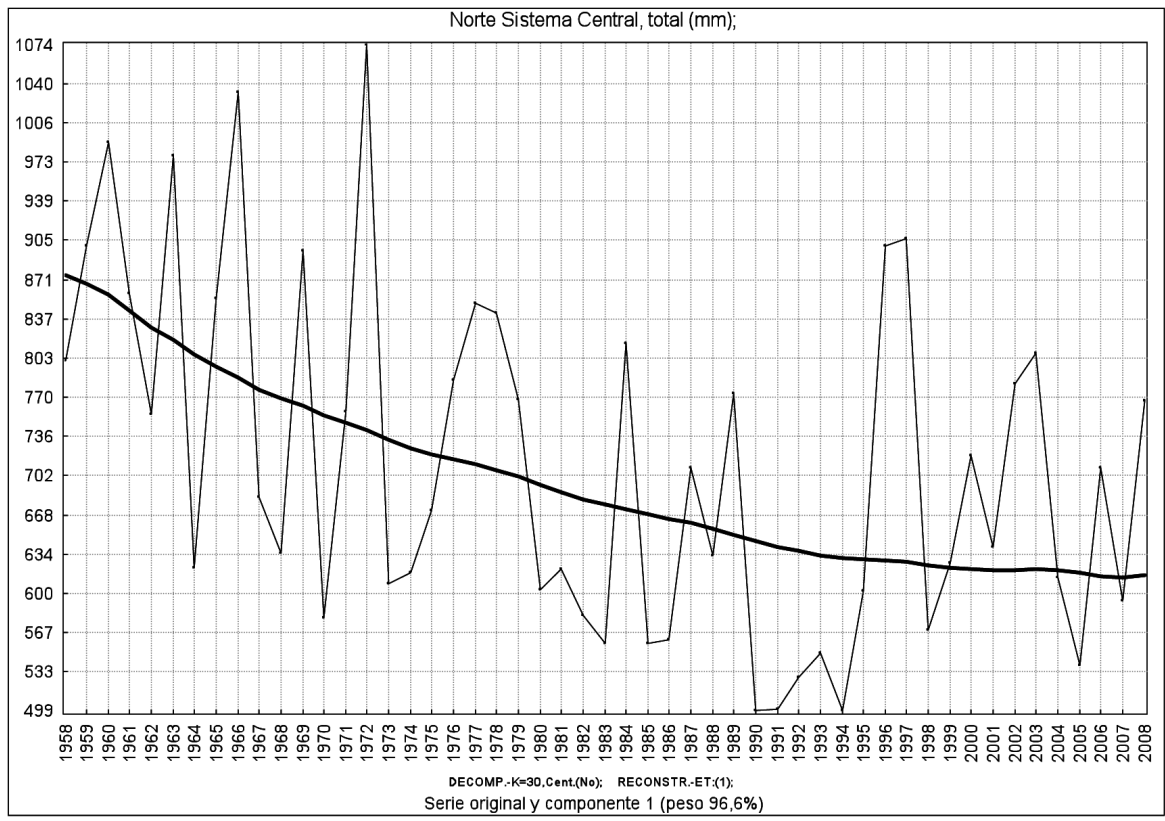

FIgURA 3. Precipitación total para el sector 'Norte del Sistema Central', y tendencia extraída mediante SSA.

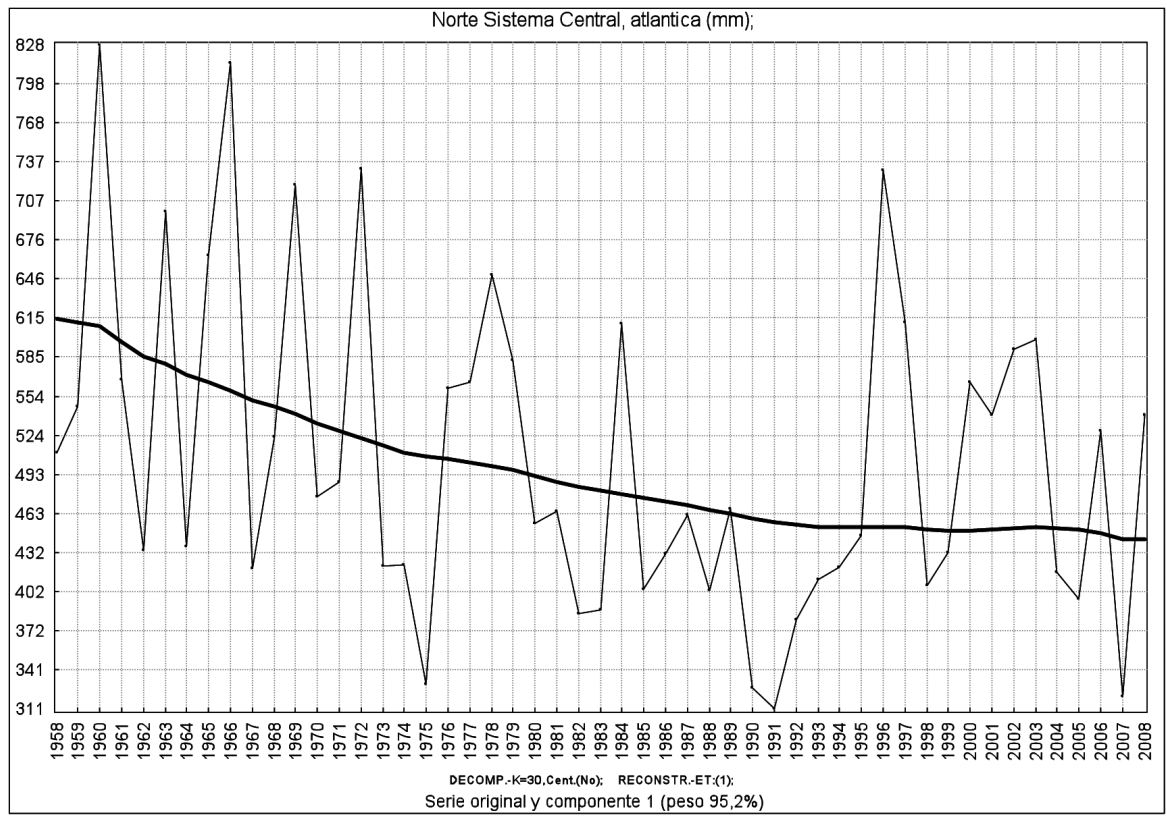

Figura 4. Precipitación por advección atlántica para el sector 'Norte del Sistema Central', y tendencia extraída mediante SSA. 


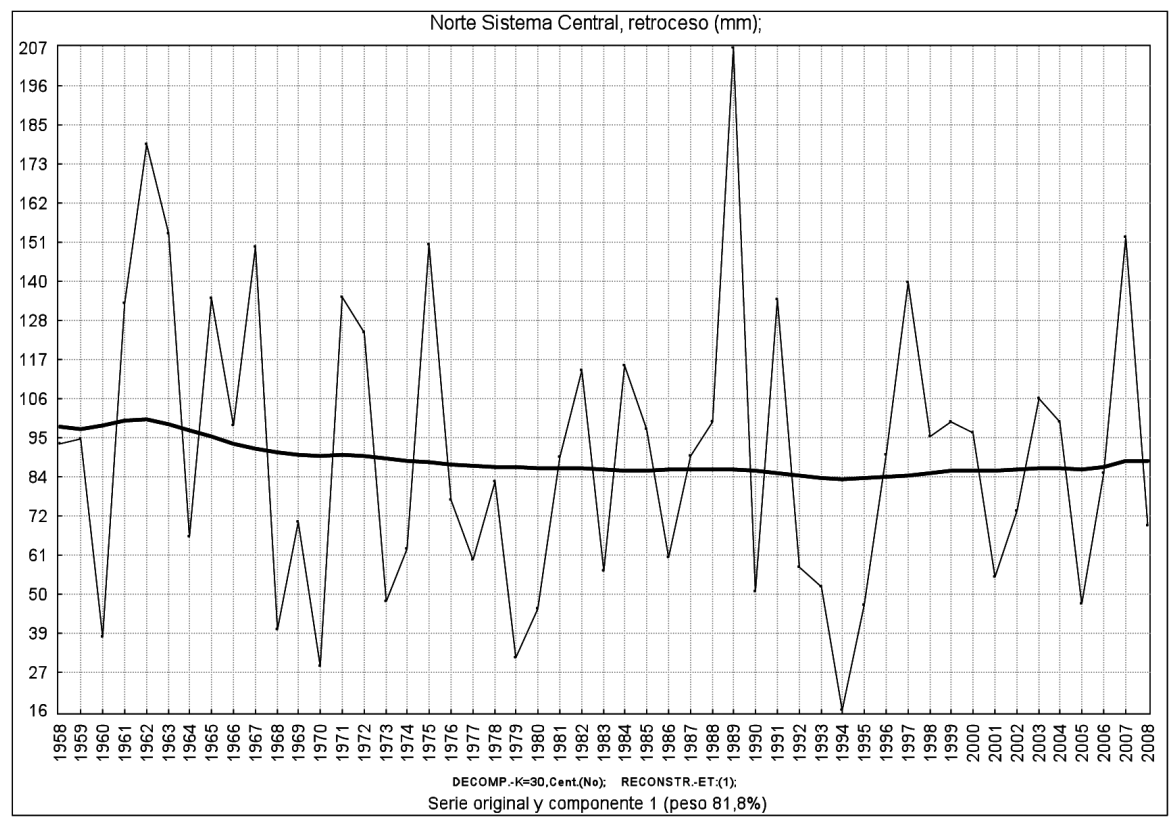

FIGURA 5. Precipitación por advección mediterránea, o de retroceso, para el sector 'Norte del Sistema Central' y tendencia extraída mediante SSA.

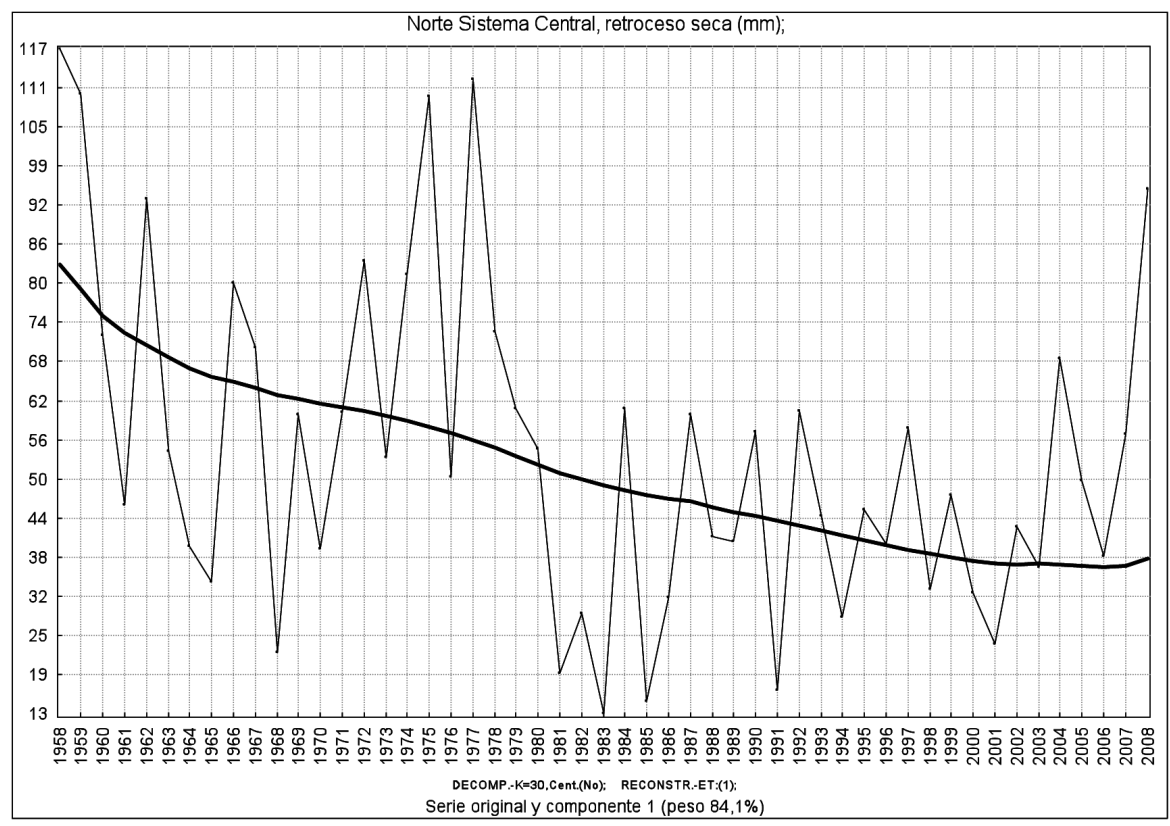

Figura 6. Precipitación por advección septentrional, o de retroceso seca, para el sector 'Norte del Sistema Central' y tendencia extraída mediante SSA. 


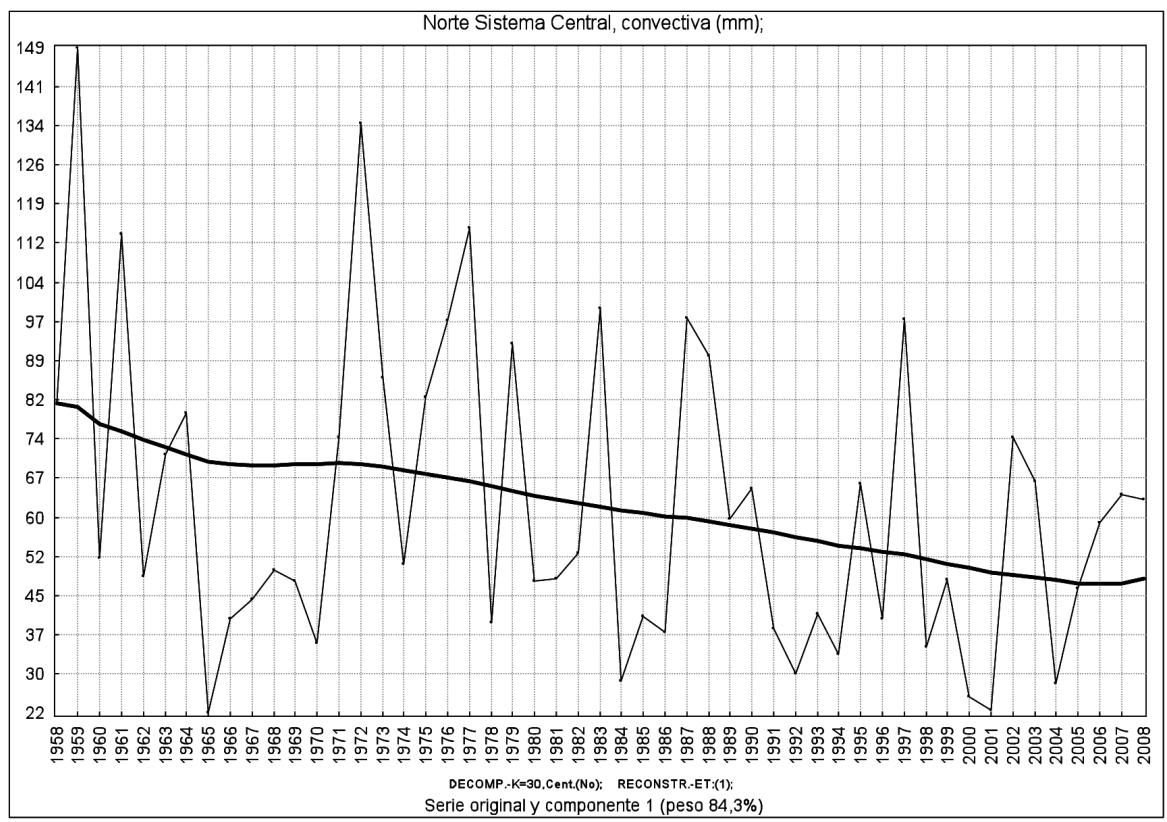

FIgURA 7. Precipitación por situaciones convectivas para el sector 'Norte del Sistema Central' y tendencia extraída mediante SSA.

y estivales. El test de Mann-Kendall muestra una tendencia negativa de casi -2, con una significación todavía aceptable superior al 90\% (Tabla 1). La estimación Sen, también en terreno negativo, puede albergar alguna duda en los intervalos de confianza, pero la línea de tendencia extraída por SSA apoya claramente la tendencia al descenso (Fig. 7).

En oposición al resto de inputs, la precipitación de 'retroceso', no presenta ninguna tendencia apreciable. Los resultados en el test de Mann-Kendall y estimación Sen no son significativos o confiables, y la línea de tendencia extraída por SSA muestra un desarrollo plano (Fig. 5). Estos resultados van en línea con los obtenidos para el mismo input en el Levante peninsular.

\subsubsection{Sector Sur del Sistema central}

Para este sector en los volúmenes totales de precipitación también se observa una tendencia negativa, aunque más moderada que la detectada para el sector al Norte del Sistema central. El test de Mann-Kendall muestra una tendencia negativa próxima a -1,7 con una significación aceptable, superior al 90\% (Tabla 1). La estimación Sen, que también da un valor negativo, deja alguna duda en los intervalos de confianza, aunque la línea de tendencia extraída por el SSA también apoya un escenario de tendencia moderadamente negativa (Fig. 8).

$\mathrm{Al}$ descender a la desagregación por inputs, se repite un comportamiento muy similar al ya observado en el sector al Norte del Sistema central, aunque mostrando un escenario general de descensos más moderados. 


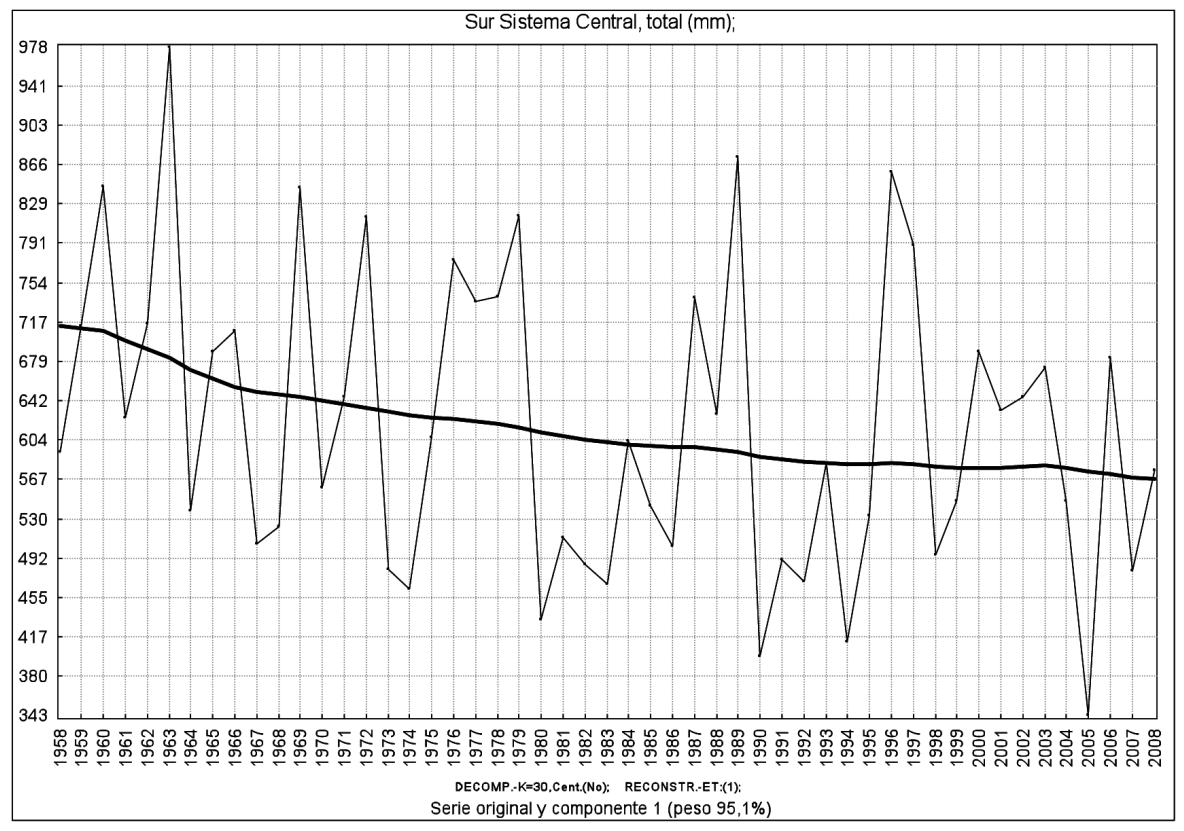

FIGURA 8. Precipitación total para el sector 'Sur del Sistema Central', y tendencia extraída mediante SSA.

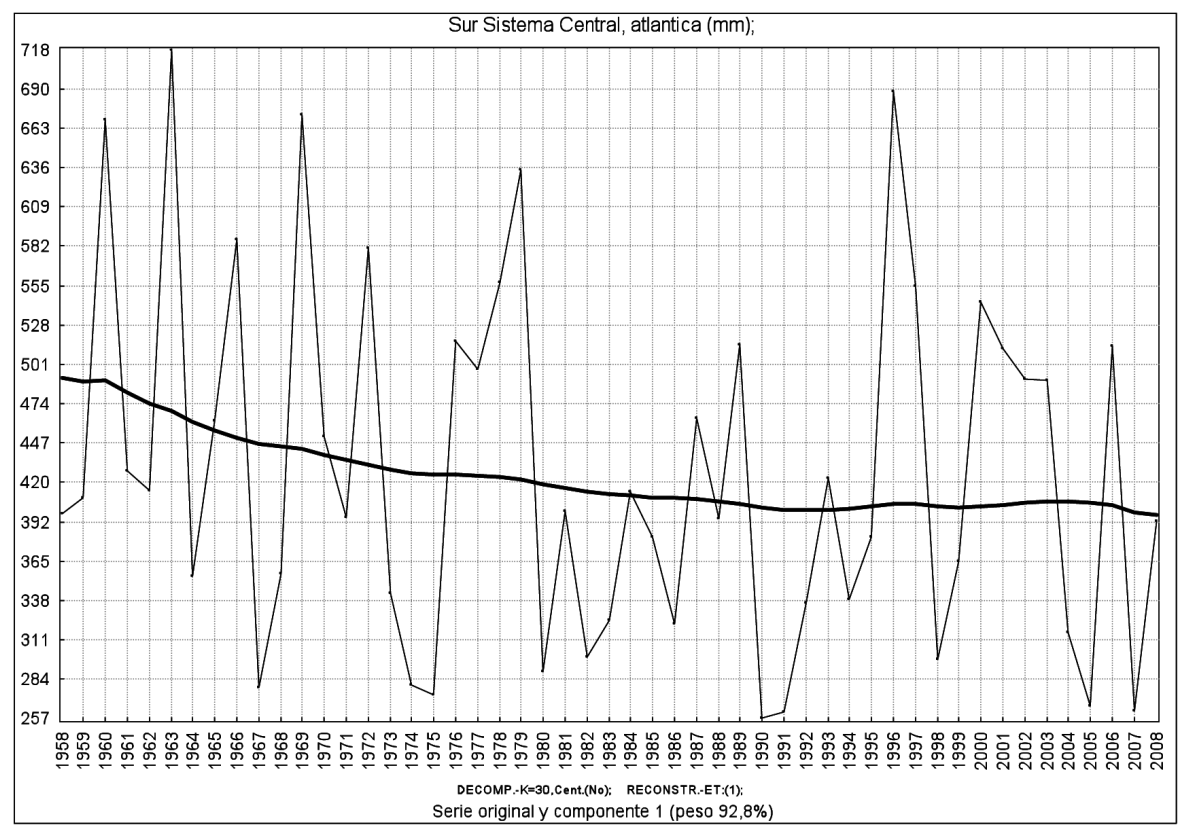

FIGURA 9. Precipitación por advección atlántica para el sector 'Sur del Sistema Central', y tendencia extraída mediante SSA. 


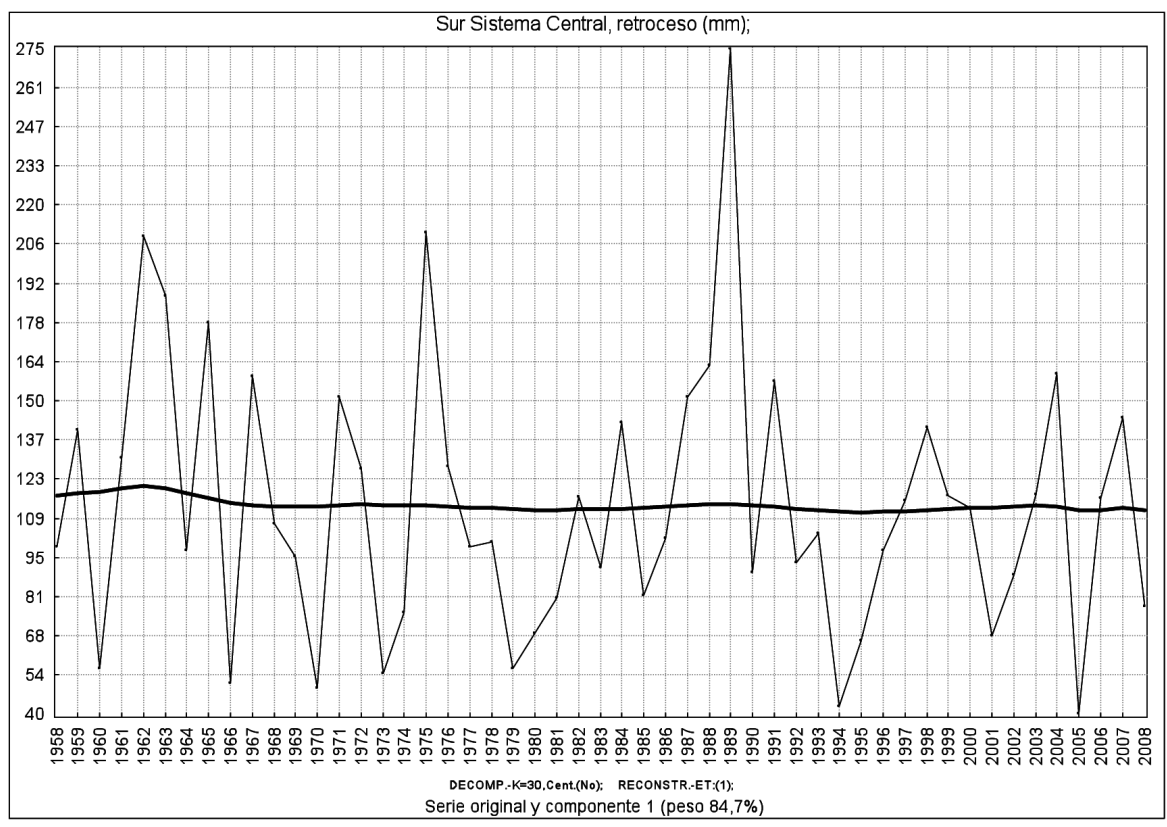

FIgURA 10. Precipitación por advección mediterránea, o de retroceso, para el sector 'Sur del Sistema Central' y tendencia extraída mediante SSA.

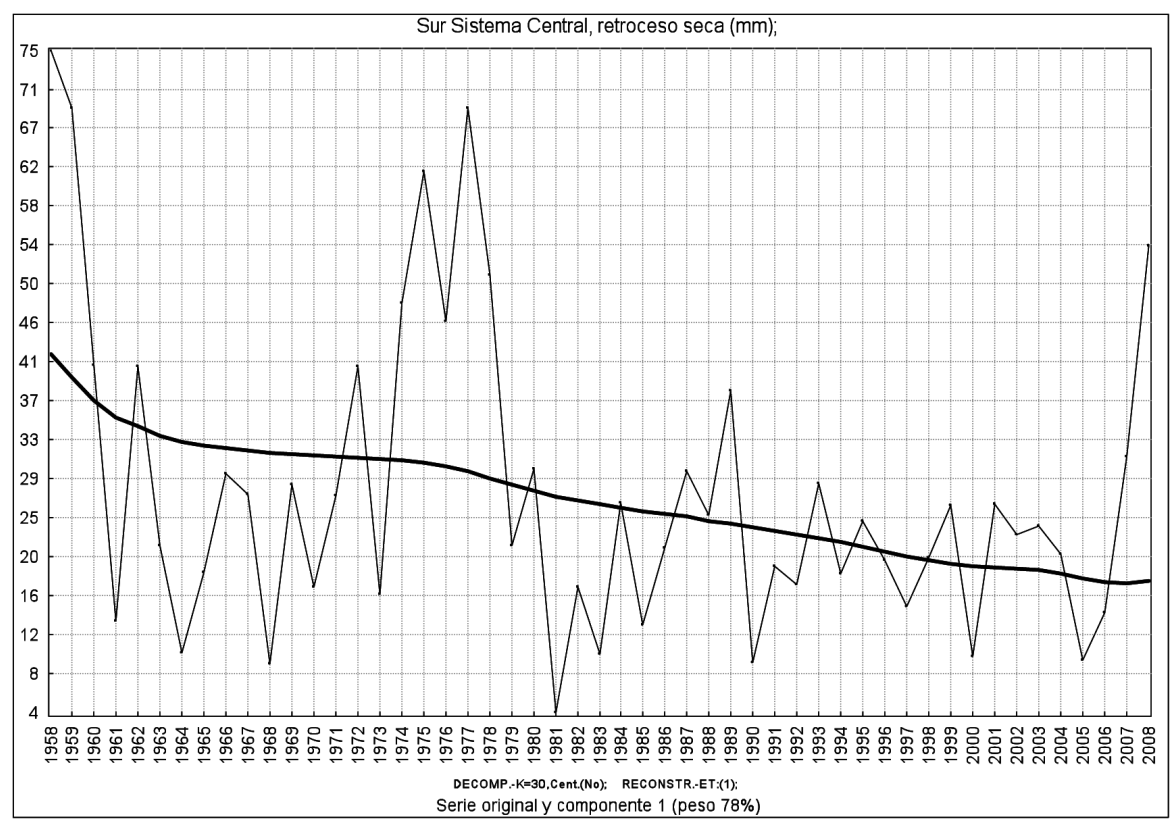

Figura 11. Precipitación por advección septentrional, o de retroceso seca, para el sector 'Sur del Sistema Central' y tendencia extraída mediante SSA. 


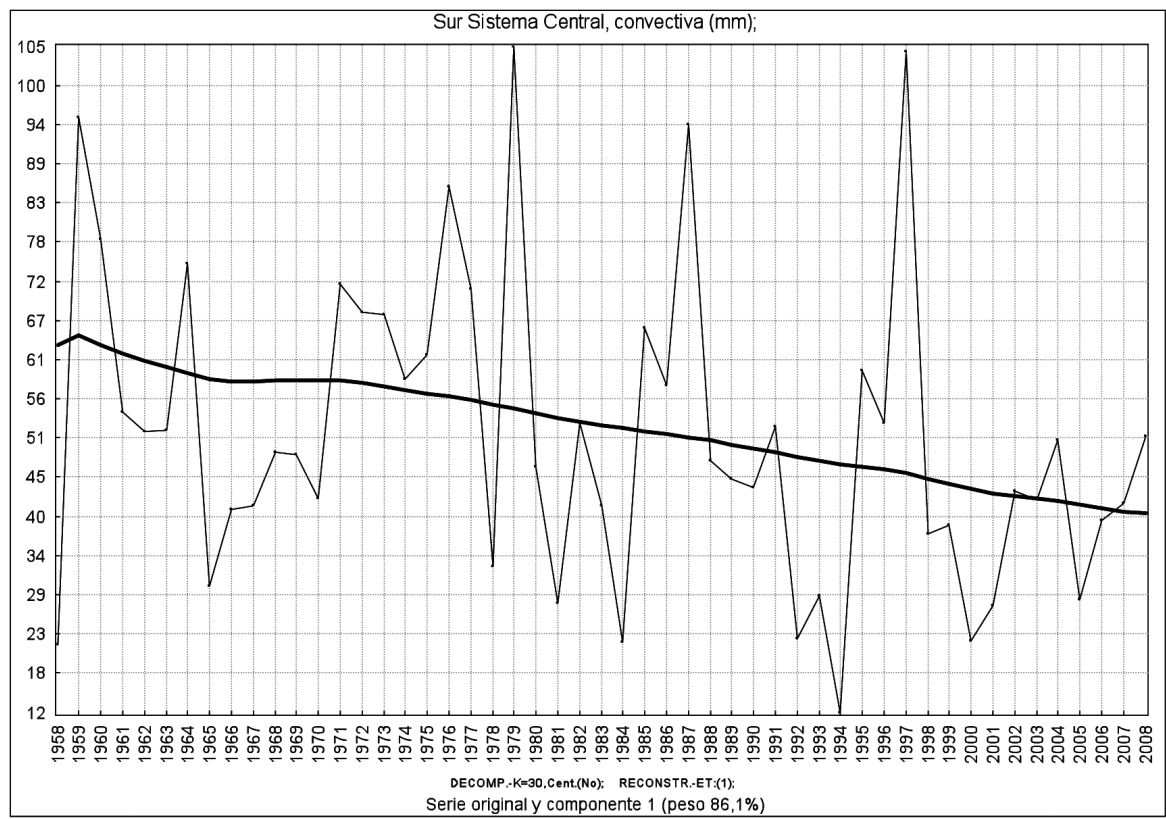

FIGURA 12. Precipitación por situaciones convectivas para el sector 'Sur del Sistema Central' y tendencia extraída mediante SSA.

Para el caso de las precipitaciones atlánticas, aunque el test de Mann-Kendall arroja un valor negativo, no alcanza a tener un buen nivel de significación. La estimación Sen de pendiente da un valor igualmente negativo, pero sin las suficientes garantías en los intervalos de confianza (Tabla 1). No obstante, la tendencia extraída mediante SSA apoya un escenario de tendencia al descenso, aunque nos revela que se produce sólo hasta inicios de los 90 (Fig. 9). Aunque tengamos aquí niveles de significación insuficientes en la tendencia al descenso, la existencia en los sectores limítrofes al Norte y Este de este sector de tendencias marcadas y confiables, aumenta las probabilidades en una tendencia al descenso, aunque menos marcada.

Respecto a las precipitaciones de tipo 'retroceso seco' y 'convectiva' sí se mantienen marcadas tendencias al descenso. El caso de las 'convectivas' es ahora más evidente, ya que el test de Mann-Kendall da un valor inferior a -2 y con un buen nivel de significación, superior al 95\% (Tabla 1). Lo apoya la estimación Sen de pendiente, con valores aceptables en los intervalos de confianza, y en mayor medida la línea de tendencia extraída por SSA, que es descendente durante todo el periodo (Fig. 12). Y en el caso del input 'retroceso seca', el test de Mann-Kendall da un valor inferior a -1.5 con un grado de significación todavía aceptable (Tabla 1). La estimación Sen también es negativa, rozando un nivel de confianza aceptable, Por último, la tendencia extraída mediante SSA para el input 'retroceso seca' es igualmente negativa desde el principio hasta el final de la serie (Fig. 11).

Al igual que ocurría en el sector Norte del Sistema Central, quedan al margen de estas tendencias negativas las advecciones de origen mediterráneo, o input 'retroceso', con unos resultados en el test de Mann-Kendall y estimación Sen poco significativos o confiables (Tabla 1), y una línea de tendencia extraída mediante SSA que muestra un comportamiento plano (Fig. 10). 


\subsection{Cambios en el peso relativo de los distintos inputs pluviométricos}

Se ha calculado el porcentaje o peso de cada input en el volumen total de la precipitación, separando en dos grupos distintos los primeros años y los últimos de toda la serie utilizada (1958-2008). Así compararemos el grupo de años de 1958 a 1978 con el grupo de años de 1988 a 2008.

En ambos sectores analizados el peso del input 'atlántica' en el volumen total de precipitación es preponderante, y próximo al 70\%. Este peso tan importante tal vez sea la razón por lo que en este sector interior peninsular la tendencia al descenso de la precipitación este muy marcada en los propios totales anuales absolutos, a diferencia de lo observado en el litoral mediterráneo. De hecho, al observar las figuras 13 y 14, se aprecia que el peso del input 'atlántica' no ha variado ostensiblemente entre los primeros 20 años de la serie y los últimos, tanto para el sector Norte del Sistema Central como para el sector Sur. Sin embargo, los inputs de menor peso como son, 'retroceso seca' y 'convectiva', sí han sufrido una significativa pérdida relativa entre ambos periodos. En el caso del sector al Norte del Sistema Central, ambos inputs pasan de representar un $9 \%$ de la precipitación a un $7 \%$ y $8 \%$ respectivamente; y en el sector al Sur, ambos pasan de un 5\% y $9 \%$ respectivamente, a un $4 \%$ y $7 \%$. Aunque sobre el $100 \%$ de la precipitación no signifique mucho, dentro de estos inputs la pérdida de peso es importante.

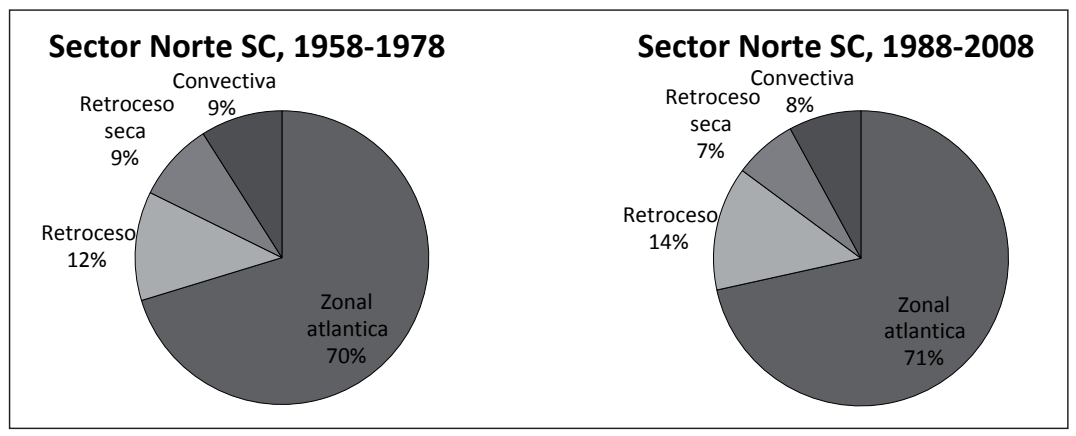

Figura 13. Sector Norte del Sistema Central. Distribución del porcentaje que representa cada input sobre el total de precipitación anual. A) Período 1958-1978. B) Período 1988-2008.

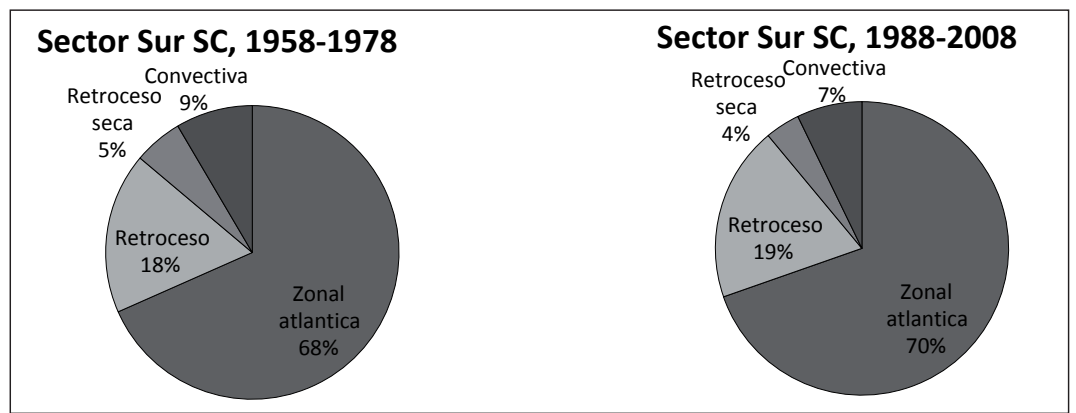

FIgURA 14. Sector Sur del Sistema Central. Distribución del porcentaje que representa cada input sobre el total de precipitación anual. A) Período 1958 1978. B) Período 1979-2008. 
Los puntos porcentuales perdidos por los inputs 'convectiva' y 'retroceso seca' se han distribuido casi por igual en los inputs 'retroceso' y 'atlántica'. Sin embargo el aumento de peso es comparativamente mayor en el caso de las advecciones mediterráneas o 'retroceso', dado que parten de tener un porcentaje mucho menor que el input 'atlántica', mientras que en éste, uno o dos puntos porcentuales más o menos, apenas suponen ningún cambio.

\section{Conclusiones}

De los resultados anteriores se puede concluir, en primer lugar, que en los últimos 50 años ha existido una tendencia al descenso de los propios totales anuales de precipitación en el sector central de la Península Ibérica. Esta tendencia es más clara y pronunciada en el sector inmediatamente al Norte del Sistema Central, mientras que es más suave en el sector al Sur del Sistema Central. También las tendencias observadas se producen principalmente entre el inicio de la serie y mediados de los años 90.

La responsabilidad de esta tendencia negativa en la precipitación recae principalmente en las advecciones de origen atlántico (input 'atlántica'), puesto que es, con mucha diferencia, el principal contribuyente al volumen total de precipitación. Sin embargo, las tendencias más acusadas al descenso se muestran en los inputs de pequeño volumen, como son 'retroceso seca' y 'convectiva', tanto al Norte como al Sur del Sistema Central.

Únicamente en las advecciones de origen mediterráneo (input retroceso) se observa un mantenimiento de los volúmenes de precipitación a lo largo de las series, no mostrando ninguna tendencia, tanto al Norte como al Sur del Sistema Central.

El hecho de tener tendencias negativas en la precipitación en todos los inputs excepto el de origen mediterráneo, sugiere que, no sólo se asiste a una pérdida del volumen total de la precipitación, sino también a un mayor peso en el comportamiento 'mediterráneo' de la misma, en línea con lo ya apuntado por otros estudios (Almarza, 2002).

Estos resultados están en consonancia con las conclusiones obtenidas con anterioridad para la zona del Levante peninsular (Estrela et al., 2004 y 2006; Millán et al., 2005 y 2006; Miró et al., 2006), y confirman la hipótesis de partida planteada por los autores de que existe una pérdida de precipitación en los sectores continentalizados de clima mediterráneo, que no se produce en el litoral mediterráneo, dada una pérdida de situaciones inestables relacionadas con circulaciones zonales a baja latitud, que no se ha manifestado en una pérdida de situaciones inestables mediterráneas (retrógradas).

Con los datos disponibles no se puede afirmar que estas tendencias sean consecuencia del cambio climático de origen antropogénico, puesto que las tendencias observadas bien podrían obedecer a ciclos naturales seculares, que durante 50 años sólo se manifiestan en forma de tendencia. No obstante, si se puede afirmar que de persistir éstas en el tiempo, el escenario de futuro estaría marcado por los descensos señalados, lo que implicaría importantes impactos en la región a medio plazo, que podrían llegar a agravar períodos de sequía.

\section{Agradecimientos}

Este trabajo ha sido financiado por el Ministerio de Educación y Ciencia a través de los proyectos GRACCIE (Programa CONSOLIDER-INGENIO 2010 CSD2007-00067), NIEVA (CGL2008-04550), y por la Comisión Europea mediante el proyecto integrado CIRCE (Proj. No. 036931-2). La Fundación CEAM está financiada por la Generalitat Valenciana y Fundación Bancaja. 


\section{Bibliografía}

ALEXANDROV, T. (2006): «Software package for automatic extraction and forecast of additive components of time series in the framework of the Caterpillar-SSA approach». PhD thesis, St.Petersburg State University.

ALEXANDROV, T.; GOLYANDINA, N. (2005): «Thresholds for methods of automatic extraction of time series trend and periodical components with the help of the «Caterpillar»-SSA approach». In: Proc. 4th Conf. System 8 Identification and Control Problems. Inst. of Control Sci, pp. 18491864, Moscow.

ALMARZA, C. (2002): «La estructura de la precipitación como índice de detección de cambio climático». Asamblea de Geodesia y Geofísica de Valencia. 4-8 Febrero 2002. Instituto Nacional de Meteorología, Madrid.

ELSNER, J. B.; TSONIS, A. A. (1996): «Singular Spectral Analysis. A New Tool in Time Series Analysis». Plenum Press.

ESTRELA, M. J.; MIRÓ, J. J.; MILLÁN, M. (2006): «Análisis de tendencia de la precipitación por situaciones convectivas en la Comunidad Valenciana (1959-2004)». En: Clima, Sociedad y Medio Ambiente. (Cuadrat, J. M., Saz, M. A., Vicente, S. M., Lanjeri, S., De Luis, M., and GonzálezHidalgo, J. C., eds.), pp. 125-136. Asociación Española de Climatología. Zaragoza.

ESTRELA, M. J.; MIRÓ, J. J.; PASTOR, F.; MILLÁN, M. (2004): «Precipitaciones por frentes atlánticos en la Comunidad Valenciana: cambios y tendencias en las últimas décadas». En: XXVIII Jornadas Científicas de la Asociación Meteorológica Española. 11-13 Febrero 2004. Badajoz.

KENDALL, M. G. (1975): «Rank correlation methods» $4^{\text {th }}$ Ed., Charles Griffin, Londres.

MANN, H. B. (1945): «Non-parametric test against trend». Econometrica, 13, pp. 245-249.

MILLÁN, M.; ESTRELA, M. J.; MIRÓ, J. J. (2005): «Rainfall components: variability and spatial distribution in a mediterranean area (Valencia Region)». Journal of Climate, 18 (14), pp. 26822705.

MILLÁN, M. M.; ESTRELA, M. J.; MIRÓ, J. J. (2006): «Análisis de tendencia de la precipitación bajo situaciones de frente de retroceso en la Comunidad Valenciana (1959-2004)». En: Clima, Sociedad y Medio Ambiente. (Cuadrat, J. M., Saz, M. A., Vicente, S. M., Lanjeri, S., De Luis, M., and González-Hidalgo, J. C., eds.), pp. 199-209. Asociación Española de Climatología. Zaragoza.

MIRÓ, J. J.; ESTRELA, M. J.; MILLÁN, M. M. (2006): «Análisis de tendencia de la precipitación por frentes atlánticos en la Comunidad Valenciana (1959-2004)». En: Clima, Sociedad y Medio Ambiente. (Cuadrat, J. M., Saz, M. A., Vicente, S. M., Lanjeri, S., De Luis, M., and GonzálezHidalgo, J. C., eds.), pp. 211-220. Asociación Española de Climatología. Zaragoza.

MIRÓ, J. J.; ESTRELA, M. J.; PASTOR., F.; MILLÁN, M. M. (2010): «Análisis comparativo de tendencias en la precipitación, por distintos inputs, entre los dominios hidrológicos del Segura y del Júcar (1958-2008)». Investigaciones Geográficas, $n^{\circ}$. Monográfico «Cambio Climático», pp. 129-157.

SALMI, T.; MÄÄTTÄ, A.; ANTTILA, P.; RUOHO-AIROLA, T.; AMNELL, T. (2002): «Makesens for detecting and estimating trends»: http://www.fmi.fi/organization/contacts_25.html

SEN, P. K. (1968): «Estimates of the regression coefficient based on Kendall's tau». Journal of the American Statistical Association. 63:1379-1389. 